\title{
Test vehicle for studying thermal conductivity of die attach adhesives for high temperature electronics
}

\author{
Conor Slater ${ }^{1, a}$, Fabrizio Vecchio ${ }^{1, b}$, Thomas Maeder ${ }^{1, b}$ and Peter Ryser ${ }^{1, d}$ \\ ${ }^{1}$ Laboratoire de Production Microtechnique, École Polytechnique Fédérale de Lausanne (EPFL), \\ Lausanne, Switzerland \\ aconor.slater@epfl.ch, ${ }^{b}$ fabrizio.vecchio@epfl.ch, ${ }^{c}$ thomas.maeder@epfl.ch, ${ }^{d}$ peter.ryser@epfl.ch,
}

Keywords: Die Attach, Thermal Conductivity, High Temperature, Electronics

\begin{abstract}
Polymer adhesives offer a viable method for mounting silicon dies for high temperature applications. Here a test vehicle for comparing the thermal conductivity of different die attach materials is presented. The setup can be used to determine the degree of degradation of polymers. It consists of a mock die that has an integrated thick film heater, which is mounted onto a substrate. In operation, the substrate is placed on a heatsink and the die is heated. When the temperature reaches equilibrium the heater is switched off and the temperature of the die is measured as it cools. The time constant of the temperature decay is calculated to give the thermal conductivity. In this paper the thermal conductivity of an epoxy die attach adhesive is compared to its shear strength.
\end{abstract}

\section{Introduction}

The trend in electronics toward devices of a reduced number of components which exclude the use of thermal management, such as cooling fans, requiring that the integrated circuits, in the form of silicon dies, run at ever higher temperatures. An example of this trend is the All Electric Aircraft. This design concept removes the hydraulic and pneumatic powered systems from the aircraft and replaces them with electrically powered systems [1]. The advantage of this is that the engines provide thrust and electrical power only, making engine design less complex and more fuelefficient. Another example is power electronics for hybrid cars [2].

Electronics that operate at temperatures exceeding $200^{\circ} \mathrm{C}$ have a unique set of problems associated with them. At these temperatures standard materials degrade over short periods of time and large temperature changes they can be subjected to can cause thermo mechanical fatigue [3]. Polymers such as silicones and epoxies can operate at these temperatures and offer resistance to fatigue due to their flexibility. Epoxies in particular are commonly used as die attach adhesives due to their high strength.

This paper presents a simple non-destructive method for evaluating the thermal conductivity of die attach adhesives. The measurement concept is presented and a mathematical model is derived that describes the behaviour of the system. The test vehicle and rig constructed to evaluate this measurement are described along with an experiment that shows that this measurement method can be used to complement traditional die attach evaluation methods such as shear testing [4].

\section{Nomenclature}

$\begin{array}{lll}Q & - & \text { Energy }(\mathrm{J}) \\ S & - & \text { Heat capacity }\left(\mathrm{J} \cdot \mathrm{K}^{-1}\right) \\ \Delta T & - & \text { Temperature difference }(\mathrm{K}) \\ L & - & \text { Adhesive thickness }(\mathrm{m}) \\ A & - & \text { Cross sectional area of adhesive }\left(\mathrm{m}^{2}\right) \\ q & - & \text { Heat flux }\left(\mathrm{W} \cdot \mathrm{m}^{-2}\right) \\ \mathrm{k} & - & \text { Thermal conductivity }\left(\mathrm{W} \cdot \mathrm{m}^{-1} \cdot \mathrm{K}^{-1}\right)\end{array}$




\section{Experimental Methods}

Figure 1 details a schematic layout of the test setup. The system consists of a mock die with an integrated PTC resistor. This is bonded to a substrate by the die attach material to be tested and the substrate is placed on a heat sink. To measure the thermal conductivity of the die attach material, the die is heated to a temperature greater than that of the substrate. Then the temperature of the die is monitored as it is allowed to cool to the temperature of the substrate. From this decay curve and the sample parameters, the thermal conductivity of the die attach material may be extracted.

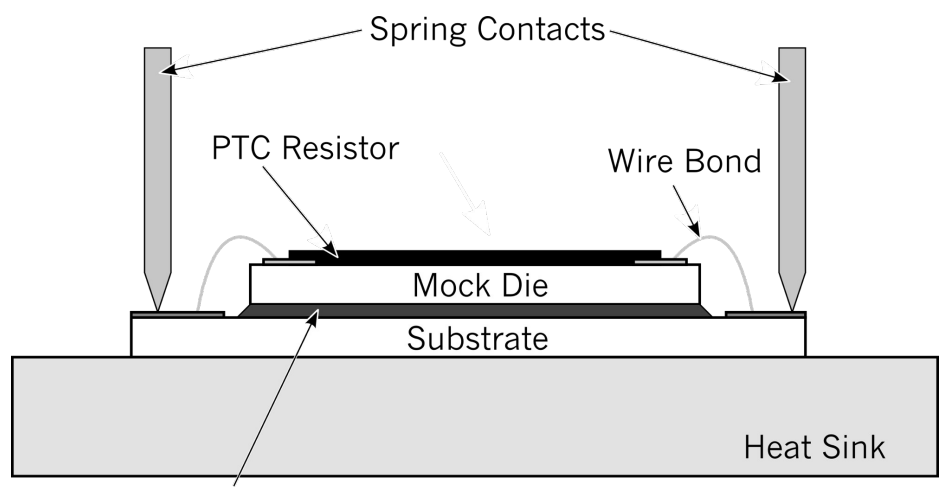

Die Attach Material

Figure 1. Schematic of test rig a test vehicle.

In this work, mock dies were manufactured from a $0.5 \mathrm{~mm}$ thick sheet of alumina. Two contact pads for each PTC resistor were screen printed with silver paste (ESL 9912-K; ElectroScience Laboratories (ESL), USA) after which the PTC paste (ESL 2611-I-SP) was printed between them. The dies were separated by scoring the alumina sheet with a laser (LS9000, Laser Systems GmbH, Germany) and breaking out the dies. The final result is a $4 \times 4 \times 0.5 \mathrm{~mm}$ die with an integrated $10 \Omega$ PTC resistor. The substrate was also screen printed with the same silver conducting paste (ESL 9912-K) to form the pads for the spring contacts and the pads for the wire bonds.

STYCAST $2741 \mathrm{~W} 1$ (Emerson and Cumming Inc., USA) was used to as the die attach material for the test vehicle. To ensure a consistent adhesive thickness the epoxy was first screen printed onto the substrate and cured for 2 hours at $65^{\circ} \mathrm{C}$. Then a second layer of the epoxy was screen printed and then 5 mock dies were placed on top using a vacuum component placer $(4907$, Dr. Tresky AG, Switzerland) to ensure that the dies were level. The assembly was then cured for 2 hours at $65^{\circ} \mathrm{C}$. After curing, the contacts on the dies were wire bonded to the silver conductors using gold wires to complete the test vehicle. The final test vehicle consisted of 5 dies attached to the substrate.

A dedicated electronics board was manufactured for the test vehicle. It could supply $600 \mathrm{~mA}$ to heat the die and $5 \mathrm{~mA}$ current source with an instrumentation amplifier to allow the resistance of the PTC to be measured. A LabJack data acquisition board (U3, Labjack Inc., USA) was used for switching between the 600 and $5 \mathrm{~mA}$ supply and for recording the voltage from the instrumentation amplifier. The entire set up was controlled by a LabVIEW program (LabVIEW 2010, National Instruments Inc., USA). 20 spring contacts were used for connecting the test vehicle to the electronics board. During experiments the test vehicle was placed on an aluminium block using heat sink compound (340, Dow-Corning Inc., USA). Figure 2 shows the test vehicle during an experiment. 


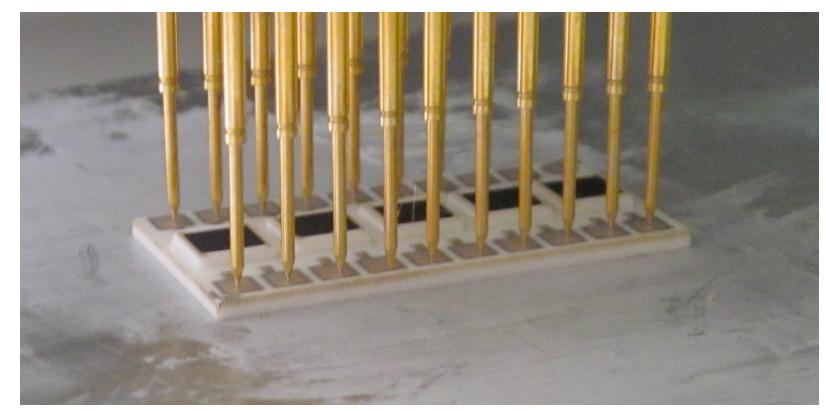

Figure 2. Test vehicle in test rig.

To measure the thermal conductivity, $5 \mathrm{~mA}$ was passed through the die under test and the voltage recorded. This was the baseline to which the voltage would return to after heating the die. Then $600 \mathrm{~mA}$ were passed through the die for a period of 2 seconds to heat it up. Subsequently the current supply was switched to $5 \mathrm{~mA}$ and the voltage across the PTC resistor was recorded at a rate of $10 \mathrm{~Hz}$. The baseline voltage was then subtracted from the recorded PTC voltage. The natural log was then calculated and a linear regression of the result was done to get the slope. The inverse of this slope was $(\tau)$ the time constant of the system.

The 5 dies on the test vehicle where evaluated by the test rig to give a time constant measurement for each die. The dies subsequently had their shear strength measured by a shear tester for comparison. Finally the surface of the sheared die was inspected under a high-magnification microscope.

\section{Data analysis}

In this work, we analyse the results using a very basic model, assuming heat transfer to be onedimensional, the temperature gradient to lie essentially across the adhesive and the heat capacity to lie essentially in the mock die (small contribution of adhesive). It is assumed that the thermal conductivity of the material of the mock die and the substrate is much greater than the die attach adhesive (i.e. the model assumes the heat is transported instantaneously to and from the die attach adhesive).

The temperature difference of the die with respect to the substrate was taken to be the amount of energy per heat capacity of the die (Eq. 1)

$$
\Delta T=\frac{Q}{S}
$$

Fourier's Law of thermal conduction is rearranged to give the temperature difference across the die attach material (Eq. 2).

$$
\Delta T=\frac{-L}{k} q
$$

The temperature difference of the die compared to the substrate is equal to the temperature difference across the die attach material thus,

$$
\frac{-L}{k} q-\frac{Q}{S}=0
$$

As the thermal flux is the amount of energy over time and area of the die attach, Eq. 3 can be rewritten as Eq. 4. 


$$
q=\frac{d Q}{d t} \therefore \int \frac{1}{Q} d Q=\frac{k A}{L S} \int d t
$$

The variables in Eq. 4 are separated and integrated. $t$ is set to 0 to find the value of constant of integration then both sides are divided by $S$ to obtain,

$$
\Delta T=\Delta T_{0} e^{\frac{-k A}{L S} t}
$$

Eq. 5 describes a system in which the differential temperature decays exponentially. The time constant $(\tau)$ is determined by the thermal conductivity of the epoxy $(k)$, the cross sectional area of the adhesive $(A)$, the adhesive thickness $(L)$ and the heat capacity $(S)$ of the die. Therefore, if the adhesive thickness and volume of the mock die are constant between samples, the thermal conductivity can be compared.

$$
\tau=\frac{k A}{L S}
$$

\section{Results and Discussion}

Figure 3 shows the output of a typical experiment. The black line shows the decay of the voltage increase across the PTC (proportional to $\Delta T$ ) after it had been heated. It decays over a period of roughly two seconds until it reaches equilibrium. The calculated natural log is shown in grey and the linear regression is shown by the black dashed line. The plots show that the decay in temperature of the system is exponential, thus the model in Eq. 5 can be considered a good approximation of the system. The inverse of the slope of the line was the time constant of the system. The time constants for the 5 dies were, $0.55,0.72,0.62,0.61$, and 0.66 seconds which is a large variation considering the same epoxy and adhesive layer thickness was used for each die.

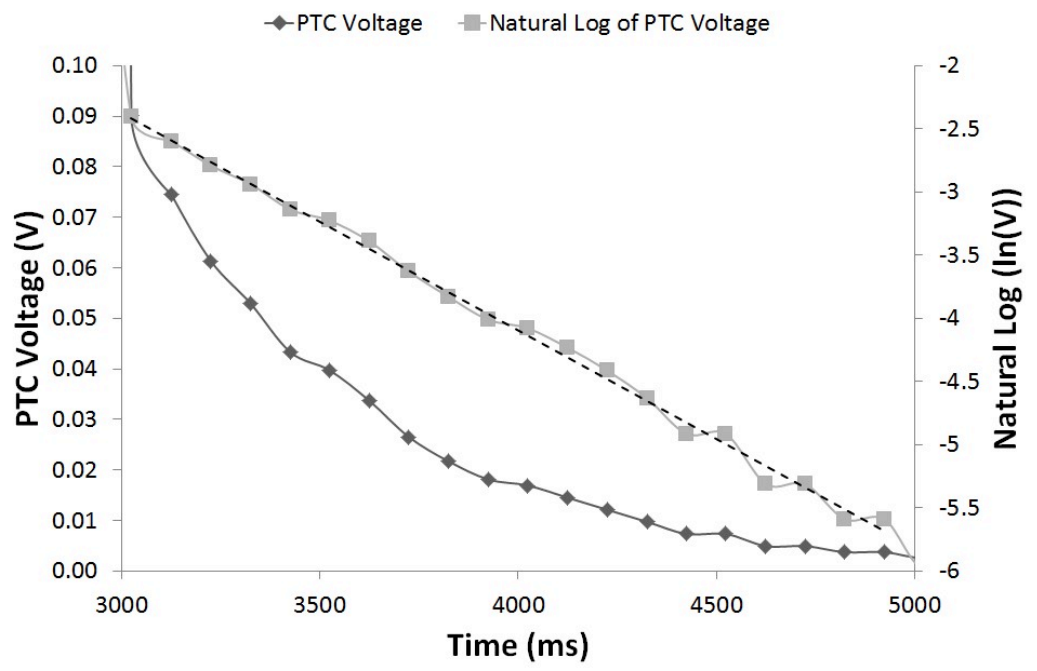

Figure 3. Plot showing exponential decay of excess PTC voltage and its natural log

The themal conductivity of the dies was calculated using an adhesive thickness of $50 \mu \mathrm{m}$ a cross sectionall area of $16 \mathrm{~mm}^{2}$ and using a heat capacity of $27.2 \times 10^{-3} \mathrm{~J} \cdot \mathrm{K}^{-1}$ (which was calculated from a $4 \times 4 \times 0.5 \mathrm{~mm}^{3}$ block of alumina with a specific heat capacity of $850 \mathrm{~J} \cdot \mathrm{Kg}^{-1} \cdot \mathrm{K}^{-1}$ ). The above time constants were used to give thermal conductivity values of $0.15,0.12,0.14,0.14$ and $0.13 \mathrm{~W} \cdot \mathrm{m}^{-}$ 
${ }^{1} \cdot \mathrm{K}^{-1}$. The thermal conductivity value of the STYCAST 2741 was reported to be $0.33 \mathrm{~W} \cdot \mathrm{m}^{-1} \cdot \mathrm{K}^{-1}$ on the datasheet published by Emmerson and Cumming.

Figure 4. shows the results of the shear test plotted against the time constants measured. One of the dies split in two while being sheared so its shear strength is omitted from the plot. The dashed line is a linear regression showing a trend in the data. It can be seen that there is a correlation between the shear strength and time constants measured.

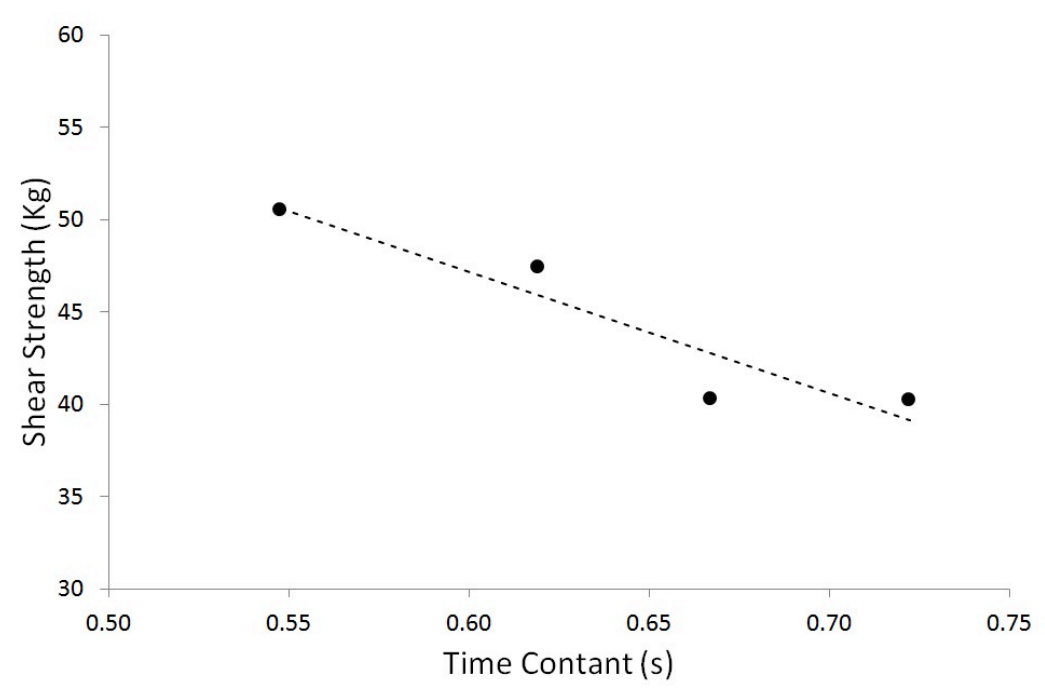

Figure 4. Plot of shear strength against time constant measurements.

Figure 5. is an image of one of the bonds on the test vehicle after it has been sheared off. The lighter areas of the die are were the epoxy has partly sheared off with the die. The dark areas are the parts of the bond that were not in contact with the base of the die (i.e. voids). Theses areas which were not in contact would have a had poor thermal conductivity as well as providing no strength to the bond. These voids were present on all the dies shear thus giving and explaination as to the correlation between the shear strength of the thermal conductivity. Furthermore the voids would have reduced the cross sectional area of the die attach thus accounting for the discrepancy in the caluclation of the thermal conductivity for the test vehicle.
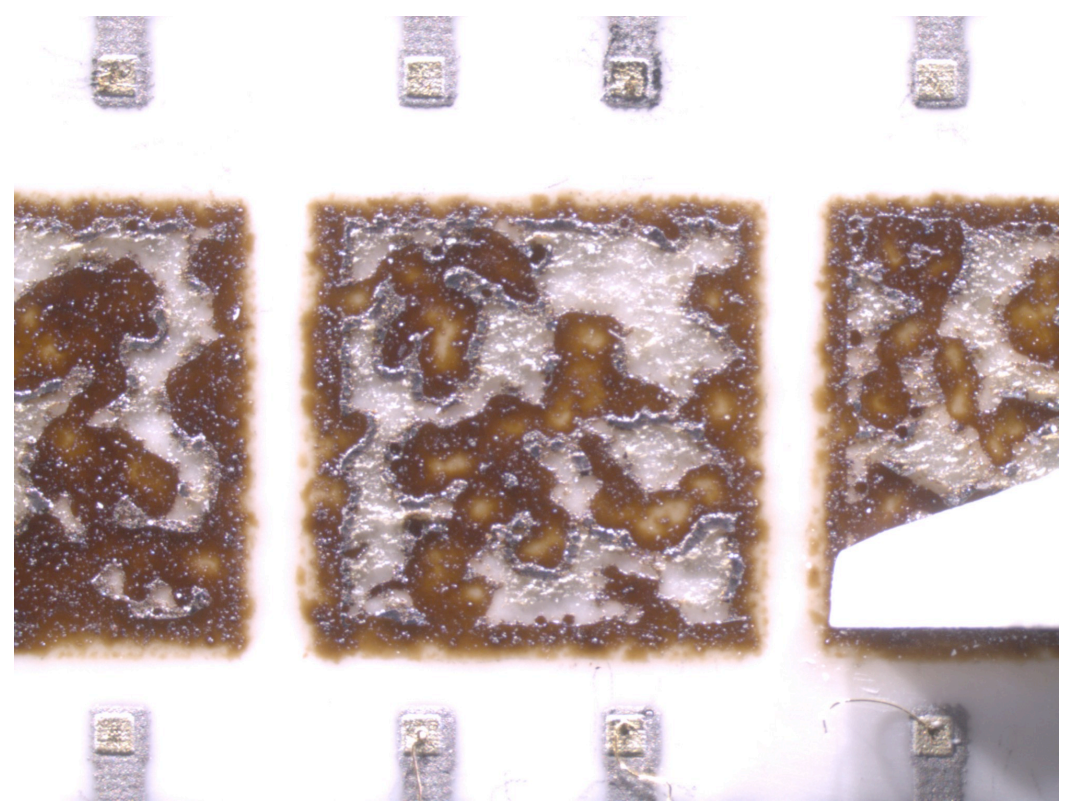

Figure 5. Epoxy on surface of test vehicle after shearing. The square of epoxy is $4 \times 4 \mathrm{~mm}$ 


\section{Conclusions}

In this article a thermal conductivity measurement concept and corresponding model were presented. This showed that the thermal conductivity, adhesive thickness and cross sectional area of the die attach, and the heat capacity of the die influenced the time constant of the temperature decay. From this a series of test vehicles were manufactured with fixed die sizes and thicknesses of the epoxy.

A test rig with dedicated electronics was constructed and the test vehicle was evaluated. The results indicated a significant variation in the time constant of the temperature decay between samples. However the analysis of the output of the test rig confirmed that the model derived was a good approximation for the system. This was further investigated by shearing the dies on the test vehicle and measuring their shear strength. When plotted a correlation between the shear strength and the time constant of the temperature decay was shown. Inspection of the sheared off dies showed voiding in the epoxy, which would have caused the thermal conductivity and the shear strength to vary. The thermal conductivity calculated using the derived of the die attach can be considered a good estimate for the die attach adhesive. It should be noted that the measurement method presented assumes that the thermal conductivity of the die attach adhesive is much less than the mock die and the substrate. Therefore measuring die attach materials with high thermal conductivity, such as solders, would not be accurate.

\section{Acknowledgement}

The research in this project has received funding from the European Union Seventh Framework Programme (FP7/2007-2013) under grant agreement ACP8-GA-2009-243119 "CREAM".

\section{References}

[1] C. Slater and P. Vassy. LTCC: A packaging technology suitable for high density integration and high temperature applications. In Proceedings of the $6^{\text {th }}$ European Aeronautics Days 2011, (In Press), 2011.

[2] B. McPherson, J. Horberger, J. Bourne, A. Lostetter, R. Schupbach, R. Shaw, B. Reese, B. Rowden, A. Mantooth, S. Ang, J. Balda, K. Okumura, and T. Otsuka. Packaging of high temperature $50 \mathrm{~kW}$ SiC motor drive modules for hybrid- electric vehicles. Advancing Microelectronics, 2010.

[3] B. Wunderle, K. F. Becker, R. Sinning, O. Wittler, R. Schacht, H. Walter, M. SchneiderRamelow, K. Halser, N. Simper, B. Michel, and H. Reichl. Thermo-mechanical reliability during technology development of power chip-on-board assemblies with encapsulation. Microsystems Technolology, 15(9):1467-1478, 2009.

[4] R. Kisiel and Z. Szczepanski. Die-attachment solutions for SiC power devices. Microelectronics Reliability, 49(6):627-629, 2009. 